\title{
Решения компании Mini-Circuits: от выбора СВЧ-компонента до производственного тестирования изделий
}

\author{
А. Бугаёв ${ }^{1}$, Ю. Ковалевский
}

\begin{abstract}
Компания Mini-Circuits, занимающая лидирующие позиции в области РЧ- и СВЧ-компонентов, в прошлом году отметила свое 50-летие. В настоящее время в ассортимент Mini-Circuits входят компоненты различного типа, включая бескорпусные монолитные интегральные схемы (МИС), миниатюрные керамические компоненты и МИС для поверхностного монтажа, модули с различными типами коаксиальных разъемов, фильтры без отражения сигнала, коаксиальные адаптеры и кабели, высокочастотные твердотельные переключатели и проч. При этом компания предлагает также решения для тестирования РЧ- и СВЧ-устройств на основе модулей собственной разработки, а также широкий инструментарий для эффективного выбора компонентов и упрощения проектирования устройств.
\end{abstract}

омпания Mini-Circuits была образована в сентябре 1968 года Харви Кэйли (Harvey Kaylie) с целью изготовления РЧ- и СВЧ-изделий со значительно меньшей стоимостью в сравнении с теми, которые предлагались на рынке на тот момент, при этом уделяя особое внимание качеству и совершенствованию процессов. Первыми результатами этой деятельности стала разработка новой линейки смесителей, трансформаторов и пассивных РЧ-компонентов, ставших успешными на международном уровне, что поспособствовало быстрому росту новой компании [1]. За годы существования компании Mini-Circuits eе продуктовая линейка выросла до таких масштабов, что способна перекрыть широкий спектр потребностей современных разработчиков и производителей высокочастотной техники, не только охватывая множество типов СВЧ-компонентов, но и предоставляя большой ассортимент средств разработки и тестирования СВЧ-электроники. И при этом линейка продукции Mini-Circuits продолжает расширяться, отвечая самым современным потребностям этой активно развивающейся области электронной техники.

Изделия СВЧ-электроники строятся на основе различных конструкторских и технологических решений, таких как волноводные, коаксиальные, микрополосковые линии, МИС, которые сами по себе могут изготавливаться на основе различных технологий, таких как арсенид- и нитридгаллиевая. Портфолио Mini-Circuits также строится на различных технологиях, о чем речь пойдет несколько позже. Вначале жехотелось бы обратить внимание на технологию

ЗАО «Золотой шар», директор по развитию. монтажа СВч-компонентов на печатные платы, что находит всё большее применение в силу продолжающейся миниатюризации и расширения функциональных возможностей электронных устройств.

\section{СВЧ-КОМПОНЕНТЫ ДЛЯ ПОВЕРХНОСТНОГО МОНТАЖА - МИЛЛИМЕТРОВЫЙ ДИАПАЗОН И ТРАДИЦИОННАЯТЕХНОЛОГИЯ СБОРКИ}

Даже оборонная промышленность стремится перейти от дорогих чипов и проводных решений ктрадиционным компонентам поверхностного монтажа на печатных платах с возможностью работы на частотах до 50 ГГц, на что обратил внимание в одном из интервью основатель компании Харви Кэйли [2]. Что уж говорить о гражданском рынке, который в последние годы активно движется в миллиметровый диапазон с развитием технологий 5 С и радарных систем в автомобилях?

В статье A Practical Design Approach to Custom mmWave SMT Packages («Практический подход к конструированию заказных корпусов поверхностного монтажа для миллиметрового диапазона»), опубликованной в мае 2019 года, приводятся результаты ожидающей патента разработки компании Mini-Circuits в области корпусов с применением в качестве материалов подложки низкотемпературной совместно спекаемой керамики (LTCC) и органических материалов. Изделия в данных корпусах продемонстрировали выдающиеся электрические характеристики при частотах до 55 ГГц [3].

Однако уже сейчас компания предлагает достаточно широкий спектр стандартных изделий в корпусах 
для поверхностного монтажа с рабочими частотами до 43,5 ГГц. В качестве примеров можно привести относительно новое изделие, предназначенное в том числе для устройств 5G, - монолитный усилитель TSS-44+ с диапазоном частот 22...43,5 ГГц и номинальной неравномерностью коэффициента усиления $\pm 0,9$ дБ в диапазоне 22...40 ГГц, выполненный в 12-выводном корпусе MCLP размером 3 ×3 мм, а также аттенюаторы серии КАТ с диапазоном частот от постоянного тока до 43,5 ГГц, большим выбором коэффициентов ослабления (от 0 до 30 дБ, причем в диапазоне от 0 до 10 дБ шаг составляет 1 дБ) и входной мощностью до 2 Вт (в зависимости от коэффициента ослабления) при том, что корпус компонента имеет размеры всего $2 \times 2$ мм.

В корпусах для поверхностного монтажа компания предлагает и другие компоненты, такие как радиочастотные смесители частоты (с диапазоном Рч до 40 ГГц), умножители частоты (с диапазоном входных частот до 20 и выходных - до 40 ГГц) и т.д.

Для разработчиков, работающих в более низких частотных диапазонах, в том числе получивших широкое распространение в потребительской, а также промышленной технике, компания предлагает широкий выбор компонентов в корпусах для поверхностного монтажа, включая генераторы с управлением напряжением, фазовые детекторы, фазовращатели, I/ Q-модуляторы и демодуляторы и др. Здесь можно упомянуть такие компоненть, как, например, усилители серий GALI и GVA с частотным диапазоном до 8 и 12 ГГц соответственно, которые выпускаются в миниатюрном корпусе SOT-89 и могут применяться в широком спектре областей, включая телекоммуникации - от оборудования базовых станций до устройств беспроводных сетей LAN, контрольно-измерительную технику, авионику и др. , а также аттенюаторы серии LAT с диапазоном частот до 2,5 ГГц в корпусах типа SOT-143.

ранее упомянутые компоненты имеют пластиковый корпус, но линейка стандартной продукции Mini-Circuits включает и изделия с повышенной защитой от внешних воздействий, такие как усилители серии СМА с различными диапазонами вплоть до 18 ГГц, корпус которых выполнен из керамики, заполнен азотом и может иметь экранирование.

Линейку компонентов для поверхностного монтажа, как и ассортимент продукции других видов, компания продолжает расширять. Среди изделий, относительно недавно дополнивших эту линейку, - компенсационный рЧ-трансформатор BLGE2-252R+, предназначенный для применения, в частности, в устройствах промышленного, научного и медицинского диапазона (ISM), а также в интерфейсах WLAN / Wi-Fi, Bluetooth и Zigbee. Компонент имеет рабочий диапазон 2,4 .. 2,5 ГГц и выполнен в корпусе 0805 из керамики LTCC.

Еще одно новое изделие - делитель/сумматор мощности SYMT-122-75+ в корпусе размером 9,65×12,7×6,35 мм для

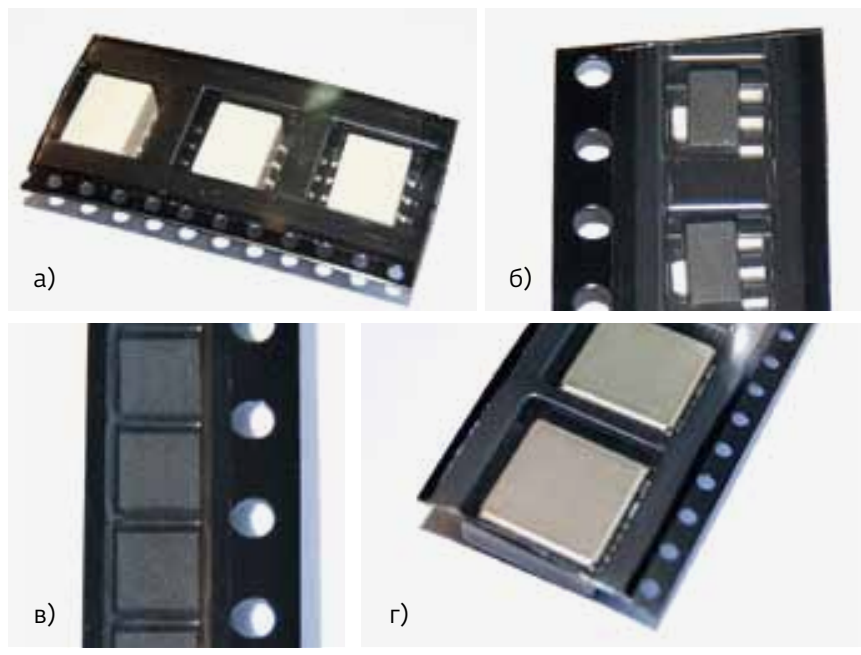

Рис. 1. Примеры типов корпусов для поверхностного монтажа РЧ- и СВЧ-компонентов Mini-Circuits: a - пластиковый корпус с выводами типа "крыло чайки" с шагом 2,54 мм; б - пластиковый корпус SOT-89; в - пластиковый корпус MCLP размером $3 \times 3$ мм; г - компонент на подложке в виде печатной платы с металлическим корпусом и зубчатыми металлизированными контактами

диапазона частот 5 ...1218 МГц с импедансом 75 Ом, который может применяться, например, в системах кабельного телевидения. Его отличительная особенность - низкий уровень несбалансированности по амплитуде (номинальное значение 0,3 дБ) и фазе $\left( \pm 3^{\circ}\right)$.

Если говорить о миниатюризации, упоминания заслуживают такие новые изделия компании, как фильтр верхних частот HPJC-492R+ для диапазона 4900...5850 МГц в корпусе 0603 и диплексор DPNK-252-492R+ для диапазонов 2400...2500 и 5150...5850 МГц в корпусе 0402.

Безусловно, упомянутые компоненты составляют лишь малую часть ассортимента компании и призваны показать, насколько широкий охват имеют изделия Mini-Circuits для поверхностного монтажа, отвечая современным потребностям в снижении стоимости и миниатюризации на фоне всё более широкого применения СВЧ-электроники и роста используемых частот в том числе в изделиях широкого применения, что обусловлено развитием технологий 5G, Интернета вещей и беспроводных систем в целом, а также новых технологий в автомобильной электронике.

\section{КРИСТАЛЛЫ МИС - ЧТО МОЖЕТ БЫТЬ МЕНЬШЕ?}

Относительная простота, дешевизна и отлаженность техпроцессов сборки методом поверхностного монтажа достаточно привлекательны для многих разработчиков и производителей. Тем не менее в определенных приложениях, например медицинских, авиационных, а также автомобильных изделиях, требуется бо́льшая защищенность от 
внешних воздействий и снижение массо-габаритныххарактеристик. Кроме того, СвЧ-изделия особенно чувствительны к длине связей и количеству и конструкции межсоединений. Поэтому в настоящее время возрастает интерес к микросборкам, микромодулям и системам в корпусе.

Помимо миниатюрных пассивных компонентов - техже, что и в технологии поверхностного монтажа, в таких изделиях применяются бескорпусные кристаллы.

Компания Mini-Circuits предлагает МИС и в таком исполнении, включая усилители, фильтры, аттенюаторы, смесители и умножители частот, делители / сумматоры мощности, ответвители и др.

у бескорпусных МИС к обозначению добавляется букBa D. B частности, одно из новых изделий, представленных компанией, - усилитель TSS-44-D+, который представляет собой бескорпусной вариант ранее упомянутого компонента TSS-44+.

\section{МОДУЛИ С КОАКСИАЛЬНЫМИ РАЗЪЕМАМИ - БЕЗ НИХ НЕ ОБОЙТИСЬ}

Несмотря на рост интереса к миниатюрным компонентам в СВЧ-электронике, они, безусловно, не могут заменить модули с коаксиальными разъемами, прежде всего в областях применения, связанных с высокими мощностными параметрами, а также - возможно, пока - очень высокими частотами.

СВЧ-компоненты всех основных типов компания MiniCircuits предлагает в модульном исполнении с различными коаксиальными разъемами, включая 1,85 мм, 2,4 мм, 2,92 мм, SMA, BNC, N-тип - в зависимости от типа компонента. Некоторые типы компонентов, такие как фильтры и умножители частоты, также имеются в исполнении, встроенном в разъем.

Усилители компании в таком исполнении охватывают диапазон частот до 44, делители мощности и аттенюаторы - до 65 ГГц.

Эта линейка включает также мощные изделия, монтируемые в стойку. Так, усилитель НРА-272+ обеспечивает выходную мощность при насыщении 100 Вт в диапазоне частот 0,7...2,7 ГГц при номинальном коэффициенте усиления 48 дБ, а усилитель HPA-100W-63+ в таком же корпусе и с такой же выходной мощностью при насыщении работает в диапазоне частот 2,5..6 ГГц, его номинальный

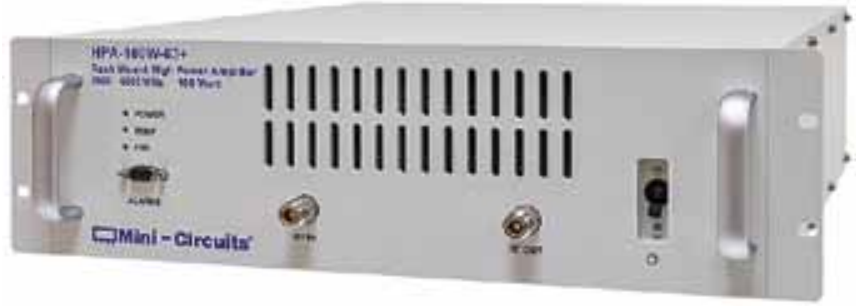

Рис. 2. Усилитель для монтажа в стойку НРA-100W-63+ [4] коэффициент усиления составляет 58 дБ. Подобные усилители в сочетании с мощными многоканальными разветвителями, такими как ZT-10HPS-272 (10 каналов, мощность 100 Вт, диапазон частот 0,7 . 2,7 ГГц) и ZT-20HPS-63 (20 каналов, мощность 100 Вт, диапазон частот 2,5..6 ГГц), также устанавливаемыми в стойку, могут применяться для выполнения тренировочных испытаний большого количества изделий на производстве.

Также линейка продукции компании включает широкий спектр адаптеров, терминаторов и других компонентов коаксиальных линий.

Заметим здесь, что теперь в ассортименте Mini-Circuits есть также и волноводные полосовые фильтры серии WVBP, охватывающие диапазон частот 27,5...86 ГГци обладающие очень низкими обратными потерями.

\section{СИСТЕМЫ ДЛЯ КОНТРОЛЯ И ИСПЫТАНИЙ - НА ЧЕМ МОЖНО СЭКОНОМИТЬ}

Решение на основе мощного усилителя и многоканального разветвителя для тренировочных испытаний, о котором говорилось ранее, - не единственное, предлагаемое компанией Mini-Circuits для выполнения различных испытаний, тестов и измерений.

В статье Innovation: The Answer to a Flat Market ("Инновации: ответ низкой активности рынка») [5] Харви Кэйли писал о том, что широко применяемые в задачах контроля и измерений анализаторы цепей обладают возможностями, во многих случаях существенно превышающими потребности пользователя, при этом затраты на них являются одними из самыхтяжелых в организации тестирования. Основными составляющими векторного или скалярного анализатора цепей для измерения отражений являются источник сигнала, детектор и высоконаправленный мост. Создав подходящий мост в виде направленных ответвителей серии ZHDC, компания Mini-Circuits предоставила своим клиентам возможность выполнять эти измерения с достаточной точностью с помощью более дешевой установки с использованием предлагаемых компанией генератора сигналов с управлением по UBS или Ethernet и датчика мощности.

Компания предлагает ряд стандартных решений для построения систем тестирования, в том числе для генерации и распределения тестовых сигналов; детектирования сигналов; инструментальные усилители; матрицы переключателей; модульные системы с различными конфигурациями переключателей, программируемыхаттенюаторов и проч., в том числе в настольном исполнении; систему для тестирования сетей с сетчатой структурой (mesh), которая может применяться для тестирования устройств Zigbee, Bluetooth, Wi-Fi и проч.

Также компания разрабатывает нестандартные станции тестирования в соответствии с требованиями конкретного заказчика. 


\section{ВСЕ ИНСТРУМЕНТЫ ОТ ПРОИЗВОДИТЕЛЯ - НА НИХ МОЖНО ПОЛОЖИТЬСЯ}

Помимо возможности сэкономить за счет применения решения, более специализированного для определенной задачи, преимуществом использования систем тестирования от производителя компонентов является то, что устраняется вероятность внесения искажений в результаты измерений, вызванных теми или иными параметрами, которые по какой-то причине либо не специфицированы производителем компонентов или тестового оборудования, либо при их измерении используются разные методики.

Безусловно, это касается только тех случаев, когда в разработке используются компоненты того же производителя, что и устройств тестовой установки. Однако, учитывая широкий спектр РЧ-и СВЧ-компонентов компании Mini-Circuits, в данном случае это обеспечить представляется вполне возможным, по крайней мере для частот от низкой до средней области миллиметрового диапазона.

По тем жепричинам возможность построения целого ряда проектов преимущественно (или полностью) на компонентах Mini-Circuits позволяет минимизировать вероятность того, что неправильное функционирование разрабатываемого изделия связано с несогласованностью компонентов, их несовместимостью из-за неких параметров, которые разные производители также могут измерять по-разному или на которые они устанавливают несколько отличные поля допусков. Таким образом значительно упрощается поиск и устранение ошибок в проектах.

Однако на этом преимущества, предоставляемые компанией Mini-Circuits для сокращения времени разработки и, как следствие, более быстрого вывода изделия на рынок, не заканчиваются. Компанией для этой цели разработан целый ряд инструментов.

Одним из них является механизм расширенного поиска компонентов по параметрам Yoni2, доступный на сайте Mini-Circuits.

Другой инструмент - калькулятор - позволяет быстро оценить такие параметры, как неравномерности коэффициента усиления и вносимых потерь, а также показатель шума двухкаскадного усилителя.

Также на сайте имеется конфигуратор систем тестирования и возможность скачать модели для симуляции ряда компонентов Mini-Circuits, разработанные совместно со специалистами компании Modelithics и совместимые с системой Keysight ADS.

Также на страницах с описанием компонентов, помимо традиционных технических описаний (datasheet), для множества изделий приводятся таблицы с данными испытаний, графики характеристик, файлы S-параметров, чертежи корпуса и упаковки (ленты для компонентов поверхностного монтажа), чертеж рекомендуемого посадочного места, схема, чертеж и Gerber-файл оценочной платы, а также параметры допустимых внешних воздействий.

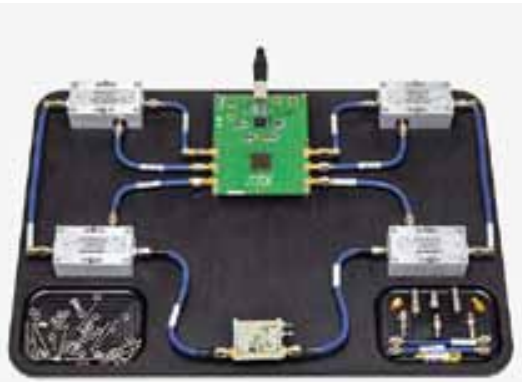

Рис. 3. UVNA-63 - набор Mini-Circuits для обучения. Позволяет построить полностью функциональный векторный анализатор цепей [6]

Наконец, компания предоставляет множество наборов для разработки, включающих компоненты определенного типа с разными параметрами. Среди них имеются наборы, содержащие усилители, делители мощности, аттенюаторы, фильтры, рч-трансформаторы и другие типы компонентов, а также коаксиальные кабели.

Таким образом, компания Mini-Circuits предлагает не только широкий спектр РЧ- и СВЧ-компонентов различной конструкции от бескорпусных кристаллов и компонентов поверхностного монтажа до мощных модулей и кабелей, но и обширный инструментарий для их выбора, симуляции проектов, прототипирования и тестирования, помогая создавать современные Рч- и СВч-изделия в кратчайшие сроки с минимальными потерями на поиск и устранение ошибок при разработке.

За более подробной информацией о продукции MiniCircuits вы можете обратиться в 3 АО «Золотой шар».

\section{ЛИТЕРАТУРА}

1. Mini-Circuits Mourns the Passing of Founder, Harvey Kaylie // Сайт Microwave Journal, 06.06.2018 // https://www.microwavejournal.com/articles/30482-minicircuits-mourns-the-passing-of-founder-harvey-kaylie

2. Kaplan B. An Interview with Mini-Circuits Founder and CEO, Harvey Kaylie // Сайт MCDI // https://www.mcdi-Itd.com/ an-interview-with-mini-circuits-founder-and-ceo-harveykaylie/

3. Gómez-Duarte C., Kahtan B., Vaisman A. A Practical Design Approach to Custom mmWave SMT Packages // Сайт Microwaves\&RF, 03.05.2019// https://www.mwrf.com/ materials/practical-design-approach-custom-mmwave-smtpackages

4. https://ww2. minicircuits.com/pdfs/HPA-100W-63+.pdf

5. Kaylie H. Innovation: The Answer to a Flat Market // Сайт MPD, 16.01.2016 // https://www.mpdigest.com/2016/01/16/ innovation-the-answer-to-a-flat-market/

6. https://www.minicircuits.com/WebStore/uvna_63.html

7. Материалы сайта https://ww2.minicircuits.com 